\title{
Ruptured aneurysm of coronary arteriovenous fistula complicated with pericardial tamponade
}

\author{
Tetsuro Uchida ${ }^{1}$, Yoshinori Kuroda ${ }^{2}$, Eiichi Ohba ${ }^{2}$, Atsushi Yamashita ${ }^{2}$, Shingo Nakai ${ }^{3}$, \\ Kimihiro Kobayashi ${ }^{4}$, Tomonori Ochiai ${ }^{1}$, and Mitsuaki Sadahiro ${ }^{3}$ \\ ${ }^{1}$ Yamagata University \\ ${ }^{2}$ Yamagata University Faculty of Medicine \\ ${ }^{3}$ Yamagata Daigaku Igakubu \\ ${ }^{4}$ Yamagata University Hospital
}

April 28, 2020

\begin{abstract}
A quarter of coronary arteriovenous fistulas may present aneurysmal dilatation; however, spontaneous rupture of the aneurysm is rare. We present a rare case of ruptured coronary artery aneurysm (CAA) associated with coronary fistulas that presented cardiac tamponade. Subsequent to pericardial drainage, surgical repair of the CAA associated with coronary fistulas was performed electively. The aneurysm was located on the left anterior aspect of the pulmonary artery trunk. It communicated with both the left and right coronary arteries by anomalous fistulous vessels that drained into the pulmonary artery trunk. The feeding arteries and fistulous opening were ligated and closed, respectively, from within the aneurysm. Intraoperative fluorescence imaging was performed pre- and postoperatively to ensure no residual coronary fistulas were present. Although the rupture of CAA associated with coronary fistulas is rare, it should be considered as one of the potential causes of acute cardiac tamponade.
\end{abstract}

\section{Introduction}

Coronary arteriovenous fistulas (CAVFs) are well-known, but are relatively rare cardiac anomalies with an occurrence of $0.002 \%$ of the general population. ${ }^{1}$ Although approximately a quarter of all CAVFs may become aneurysmal, its spontaneous rupture with CAVFs is rare. ${ }^{2-6}$ Rupture of coronary aneurysm can be lethal because of cardiac tamponade. Herein, we discuss a successful surgical case of ruptured coronary artery aneurysm (CAA) associated with CAVFs presenting with cardiac tamponade.

\section{Case Report}

A 48-year-old woman was referred to our institution for surgical management of ruptured CAA associated with CAVFs. Four days before referral, she had experienced chest discomfort followed by presyncope. Following medical examination at the neighboring hospital, her condition was diagnosed as cardiac tamponade due to rupture of the CAA with CAVFs. Computed tomography (CT) scan revealed an aneurysmal formation measuring $3.0 \times 2.0 \mathrm{~cm}$, which was located at the left anterior aspect of the pulmonary artery trunk with a large amount of pericardial effusion (Fig. 1a). Pericardial drainage was performed and $100 \mathrm{~mL}$ of blood was aspirated (Fig. 1b). Subsequently, her symptom improved immediately. Additionally, CT scan revealed that the aneurysm was communicated with the left and right coronary arteries (RCA) through anomalous fistulous vessels that drained into the pulmonary artery trunk (Fig. 1c). Fistulous vessels originating from proximal RCA and left anterior descending artery (LAD) were confirmed by coronary angiography. With the diagnosis of ruptured CAA with CAVFs, operation was urgently performed. 
The patient underwent a median sternotomy. Surgical exposure revealed mild fibrous adhesion mainly around the pulmonary artery and a saccular-type mass measuring $3.0 \mathrm{~cm}$ in diameter at the left anterior surface of the pulmonary artery (Fig. 2a). Prior to repair of the CAVFs, intraoperative near-infrared fluorescence imaging of CAVFs was performed using photodynamic eye (Hamamatsu Photonics, Hamamatsu, Japan). ${ }^{7}$ Approximately 15 seconds after intravenous injection of indocyanine green, abnormal fistulous vessels were visualized at the anterior surface of the pulmonary artery (Fig. 3a, 3b). Cardiopulmonary bypass was established between the ascending aorta and bicaval cannulation. Following aortic cross clamping, cardiac arrest was achieved by antegrade cardioplegia. First, the feeding arteries originating from the proximal RCA were ligated at their origin. Second, the pulmonary artery was incised longitudinally and the fistulous opening that communicated between the pulmonary artery and aneurysm was closed with a pledgeted suture (Fig. $2 \mathrm{~b})$. The CAA was opened and the drainage sites from the feeding arteries were identified by antegrade infusion of cardioplegia (Fig. 2c). These drainage sites were closed from within the aneurysm followed by aneurysmorrhaphy. Weaning from the cardiopulmonary bypass was uneventful. After completion of surgical repair, no residual CAVFs was confirmed from repeated fluorescence imaging (Fig. 3c). The patient's postoperative recovery was uneventful and she was discharged 7 days after surgery. Postoperative CT did not reveal any evidence of residual fistulas. Recurrent CAVFs and CAA were not observed during follow-up. Written informed consent was obtained from the patient for the publication of this report.

\section{Discussion}

Aneurysmal change of CAVFs is common and $19-26 \%$ of CAVFs are complicated with saccular type CAA ${ }^{8}$; however spontaneous rupture of aneurysm is rare. Treatment of asymptomatic patients with CAA associated with CAVFs is still debated given its rarity. CAAs exceeding $30 \mathrm{~mm}$ in diameter are considered to be at the risk of rupture ${ }^{9}$; however, there are reports of rupture in cases of aneurysms measuring $10 \mathrm{~mm}$ and $15 \mathrm{~mm} .{ }^{10,11}$ Therefore, surgical intervention should be considered for coronary aneurysm of any size with CAVFs to prevent rupture of the aneurysm.

Cardiac tamponade with hemodynamic deterioration is often caused by acute aortic dissection, free wall rupture due to acute myocardial infarction, or a traumatic thoracic aortic injury. Some reports, including our report, have described ruptured CAA with CAVFs resulting in cardiac tamponade. ${ }^{1-13}$ In fact, there has been a report of emergency surgery without a definitive diagnosis. ${ }^{12}$ Therefore, rupture of CAA with CAVFs should be considered as one of the potential causes of acute cardiac tamponade.

Although prognosis after successful surgical closure of CAVFs is excellent even in ruptured cases, long-term follow-up is essential, because residual CAVFs and recurrences are reported with considerable frequency. ${ }^{14}$ Residual and late recurrence of CAVFs are considered to result from incomplete surgical procedure. ${ }^{14}$ To avoid these complications, it is important to ensure the absence of residual CAVFs. Fluorescence imaging can furnish sharp images with notable spatial and temporal resolution. Intraoperative fluorescence imaging is a safe, non-invasive, and useful diagnostic tool for the identification of residual CAVFs. ${ }^{7}$ The resulting images enable easy detection of residual CAVFs in real time.

\section{Author Contributions:}

TU has a major contribution in writing the manuscript.

\section{References}

1. Dodge-Khatami A, Mavroudis C, Backer CL. Congenital heart surgery nomenclature and database project: anomalies of the coronary arteries. Ann Thorac Surg 2000;69:S270-297.

2. Said SA, el Gamal MI, van der Werf T. Coronary arteriovenous fistulas: collective review and management of six new cases-changing etiology, presentation, and treatment strategy. Clin Cardiol 1997;20:748-752.

3. Sugiyama K, Suzuki S, Kamiya K, Koizumi N, Ogino H. Ruptured coronary artery aneurysm with pulmonary artery fistulae. J Card Surg 2017;32:799-800. 
4. Uzuka T, Nakamura M, Nakajima T, Watanabe N, Fukazawa Y. Surgery of giant right coronary artery aneurysm complicated with coronary artery fistula to left ventricle. J Card Surg 2018;33:95-96.

5. Kumagai M, Takatoku K, Kawamoto A, Shinoda E, Nishizawa J. Giant coronary artery aneurysm with a coronary artery fistula to the pulmonary artery. J Card Surg 2018;33:131-132.

6. Uchida T, Hamasaki A, Kuroda Y, Ohba E, Yamashita A, Sadahiro M. Novel surgical technique for coronary fistulas with proximal origin. Ann Thorac Surg 2017;104:e261-e263.

7. Uchida T, Hamasaki A, Kuroda Y, Yamashita A, Sadahiro M. Surgical management of proximal coronary arteriovenous fistulas using intraoperative fluorescence imaging. J Card Surg 2018;33:836-839.

8. Urrutia-S CO, Falaschi G, Ott DA, Cooley DA. Surgical management of 56 patients with congenital coronary artery fistulas. Ann Thorac Surg 1983;35:300-307.

9. Takeuchi N, Takeda M, Nishibori Y, Maruyama T. A case report of coronary arteriovenous fistulas with an unruptured coronary artery aneurysm successfully treated by surgery. Case Rep Cardiol 2012;314685:1-6.

10. Sakao T, Tsunooka N, Nakagawa H, Kajiwara S. Ruptured saccular aneurysm of a coronary artery to pulmonary artery fistula associated with cardiac tamponade. Kyoubu Geka 2000;53:684-686.

11. Sakamoto S, Kanemitsu S, Shimpo H, Kurita T. Tiny ruptured coronary aneurysm with coronarypulmonary arterial fistula. Gen Thorac Cardiovasc Surg 2019;67:1084-1086.

12. Kawano M, Wada T, Anai H, Shuto T, Miyamoto S. A case of ruptured aneurysm of coronary-pulmonary artery fistula diagnosed after emergency thoracotomy. Surg Case Rep 2018;4:24.

13. Shomura Y, Mizumoto T, Fujinaga K, Sawada Y, Ito H, Teranishi S. Cardiac tamponade due to rupture of a giant coronary artery aneurysm with a coronary arteruivenous fistula: a case report. Surg Case Rep 2019;5:40.

14. Cheung DL, Au WK, Cheung HH, Chiu CS, Lee WT. Coronary artery fistulas: long-term results of surgical correction. Ann Thorac Surg 2001;71:190-195.

Figure Legends

Figure 1: CT images of CAA associated with coronary fistulas.

a) Coronary artery aneurysm measuring $3.0 \times 2.0 \mathrm{~cm}$ located at the left-anterior aspect of the pulmonary artery trunk (white arrow). b) Pericardial effusion is noted. c) Three-dimensional CT image showing CAA associated with coronary fistulas (white arrowheads).

$A o$, ascending aorta; $A n$, aneurysm; $P A$, pulmonary artery; $R A$, right atrium; $R V$, right ventricle

Figure 2: Intraoperative photographs.

a) CAA is located at lateral surface of the pulmonary trunk (dotted area). b) Fistulous opening which communicates the pulmonary artery and coronary aneurysm is observed (black arrow). c) From within the aneurysm, drainage site of feeding arteries is closed (white arrow).

$A o$, ascending aorta; $A n$, aneurysm; $P A$, pulmonary artery; $R A$, right atrium; $R V$, right ventricle

Figure 3: Intraoperative fluorescence imaging.

a, b) Preoperative images showing anomalous fistulous vessels in front of the pulmonary artery (white arrows). c)Postoperative image showing ligated feeding arteries arising from the proximal right coronary artery (black arrow). Anomalous fistulous vessels and CAA are not visualized.

$A o$, ascending aorta; $A n$, aneurysm; $P A$, pulmonary artery; $R A$, right atrium; $R V$, right ventricle

\section{Hosted file}


Figure.pptx available at https : //authorea.com/users/312766/articles/443360-ruptured-aneurysmof-coronary-arteriovenous-fistula-complicated-with-pericardial-tamponade 